\title{
Pushing the Limits of Microfocus X-Ray Sealed Tube Sources for Crystallography
}

\author{
J. Graf ${ }^{1}$, T. Stuerzer ${ }^{2}$, M. Benning ${ }^{3}$, R. Durst ${ }^{2}$, P. Radcliffe ${ }^{1}$, C. Michaelsen ${ }^{1}$ \\ ${ }^{1}$ Incoatec GmbH, Geesthacht, Germany, ${ }^{2}$ Bruker AXS GmbH, Karlsruhe, Germany, ${ }^{3}$ Bruker AXS LLC, Madison WI, USA \\ graf@incoatec.de
}

The structure determination on ever smaller and weakly diffracting crystals is one of the biggest challenges in the development of inhouse X-ray analytical equipment for chemical and biological crystallography, which continuously raises the requirements for modern X-ray sources and detectors. Nowadays, modern low power microfocus X-ray sealed tube sources define the state-of-theart for most in-house X-ray diffraction equipment, as they deliver intensities in the range of rotating anodes, yet maintain all the comfort of a sealed tube system.

Throughout the past years, we have continuously explored the physical limitations of impact ionization sources in order to find ways to push or even overcome some of the limitations, such as the heat transfer in the anode, leading to brighter X-rays sources with solid targets. The brightness of an X-ray tube is mainly limited by the thermal conductivity of the bulk anode material. As the thermal conductivity of diamond is up to about 5 times higher than that of copper and the highest known conductivity of all bulk materials [1], industrial diamond is increasingly replacing traditional materials for the thermal management in challenging applications [2], in which a high local heat load needs to be dissipated, such as in heat sinks for high-power microelectronic devices [3, 4]. In X-ray sources, diamond can be used as a heat sink directly coupled to the anode material, resulting in a significantly higher thermal conductivity compared to a conventional metallic anode and, hence, allowing for an increase in tube brilliance by applying a higher power load on the anode [5].

As a result of our efforts, we recently introduced a unique new class of microfocus sealed tube X-ray sources that uses a novel anode technology, the diamond hybrid anode [6]. It consists of a thin layer of metal deposited onto a bulk industrial diamond which acts as a heat spreader and significantly improves the heat dissipation in the anode. Consequently, the anode can accept a higher power density in the focal spot on the target without damaging the surface of the target layer. The balanced heat management allows the source to be aircooled, while assuring that the intensity loss over time is only a few percent over $10,000 \mathrm{~h}$ of full power operation, which is significantly lower than the intensity degradation observed for microfocus rotating anodes [7, 8]. Along with this, optimizing the takeoff angle of the anode and the filament parameters of the cathode to match the requirements of the X-ray optics enables a significant increase in the intensity on the sample. In combination with the latest developments in multilayer mirror technology, the $\mathrm{I} \mu \mathrm{S}$ delivers an intensity in the range of $1 \cdot 10^{11} \mathrm{phts} / \mathrm{s} / \mathrm{mm}^{2}$ with a divergence that matches the typical mosaicity of weakly diffracting samples. Therefore, the I $\mu \mathrm{S}$ DIAMOND combines the performance of a modern $1 \mathrm{~kW}$ microfocus rotating anode with all the comfort of a conventional microfocus sealed tube source.

We will be reviewing the latest innovations in microfocus sealed tube X-ray sources and multilayer optics and be presenting selected results from protein and pharmaceutical crystallography that demonstrate the impact of these recent developments on the data quality.

[1] Moore, A.L. \& Shi L. (2014). Materials Today 17, 163.

[2] Dischler B. \& Wild C. (1998). Low-Pressure Synthetic Diamond Manufacturing and Applications. Berlin: Springer.

[3] Obeloer T., Bolliger B., Han Y., Long Lau B., Tang G. \& Zhang X. (2015). IMAPS, 1.

[4] Pu S., Luo W., Shuai Y., Wu C. \& Zhang W. (2016). ICMIA, 184.

[5] Li X., Wang X., Li Y. \& Liu Y. (2020). Materials 13, 241.

[6] Durst R. D., Michaelsen C., Radcliffe P. \& Schmidt-May J. (2020). US Patent 10,847,336.

[7] Mehranian A., Ay M. R., Riyahi Alam N. \& Zaidi H. (2010). Med. Phys. 37, 742.

[8] Kákonyi R., Erdélyi M. \& Szabó G. (2010). Med. Phys. 37, 5737.

Keywords: Diamond, Crystallography, X-ray Instrumentation 